\title{
Performance of Asymmetric Digital Subscriber Lines in an Impulse Noise Environment
}

\author{
Wei Yu, Dimitris Toumpakaris, John M. Cioffi, Daniel Gardan, and Frédéric Gauthier
}

\begin{abstract}
This letter presents a numerical study of the impact of impulse noise on asymmetric digital subscriber lines (ADSL). Methods for simulating the effect of impulse disturbances on a discrete multitone system are first presented, and actual measured noise bursts are then used for the simulations as if they were deterministic signals, in order to characterize their effects on ADSL systems. It is shown that while a combination of coding, interleaving, and 6-dB margin is adequate in protecting ADSL systems from isolated impulses, an impulse train with long duration will cause a significant number of error bits in the system. In this case, a tradeoff among the number of error seconds, the maximum reach, and the coding delay must be made.
\end{abstract}

Index Terms-Asymmetric digital subscriber lines (ADSL), discrete multitone (DMT), forward error correction (FEC), impulse noise, Reed-Solomon (RS) codes.

\section{INTRODUCTION}

$\mathbf{O}$ CCASIONALLY, large nonstationary electromagnetic disturbances may be coupled into telephone wires, resulting in impulse noise. The objective of this paper is to present a study of the impact of impulse noise on asymmetric digital subscriber line (ADSL) systems. The study is carried out in two steps. First, the effect that a deterministic time-domain impulse has on the performance of a multicarrier system is examined, and a method to accurately simulate the performance of a discrete multitone (DMT)-based G.dmt-compliant [1] ADSL system is described. Methods to simulate the effect of impulse noise on a system with Reed-Solomon (RS) codes do not appear to have been presented in the open literature, to the best of the authors' knowledge. Second, realistic impulses as measured by France Télécom are used to simulate both uncoded and RS-coded ADSL systems. The performance is characterized in terms of both the probability of bit error and the number of error bytes for isolated impulses, and in terms of the number of error seconds for impulse trains.

Previous studies in this area [2]-[6] mostly rely on statistical models of the impulse noise. This letter recognizes that the ac-

Paper approved by C.-L. Wang, the Editor for Equalization of the IEEE Communications Society. Manuscript received June 14, 2002; revised November 15, 2002 and April 11, 2003.

$\mathrm{W}$. $\mathrm{Yu}$ is with the Electrical and Computer Engineering Department, University of Toronto, Toronto, ON M5S 3G4, Canada (e-mail: weiyu@ comm.utoronto.ca).

D. Toumpakaris was with the Department of Electrical Engineering, Stanford University, Stanford, CA 94305 USA. He is now with Marvell Semiconductor, Inc., Sunnyvale, CA 94085 USA (e-mail: dimitris@ ieee.org).

J. M. Cioffi is with the Department of Electrical Engineering, Stanford University, Stanford, CA 94305 USA (e-mail: cioffi@ stanford.edu).

D. Gardan and F. Gauthier are with France Télécom R\&D, 22307 Lannion, France (e-mail: daniel.gardan@ francetelecom.com; frederic.gauthier@ francetelecom.com).

Digital Object Identifier 10.1109/TCOMM.2003.818107 curate modeling of impulse noise is not an easy task, and instead relies on measured impulses to study the behavior of an actual system in a real environment. As it is expected and will be verified by the simulations, the impact of impulse noise on a practical ADSL system depends strongly on the impulse amplitude, its duration, the interarrival time, and the spectral characteristics of the impulse.

\section{ADSL Performance IN GAUSSIAN NoISE}

This section presents a method to compute the probability of bit error in a multicarrier system with RS-based forward error correction (FEC) in an additive white Gaussian noise (AWGN) environment. Specific values are used to simplify the description of the method.

Assuming an RS codeword of 200 bytes, 16 bytes of which are parity, for an RS codeword to be correctable, at most, eight bytes can be in error. It is well known [7] that the miscorrection probability of RS codes is very small, and it will be ignored in the following calculations. In an AWGN or properly equalized channel, the probability of error $P_{\text {byte }}$ for each byte is approximately the same. When $P_{\text {byte }}$ is small, as in typical ADSL deployments, the probability of codeword error is closely approximated by the probability that nine erroneous bytes occur, as events with eight or fewer errors are corrected by the code, and events with more than nine errors occur with much lower probability. Hence, the probability of codeword error is approximately

$$
\left(\begin{array}{c}
200 \\
9
\end{array}\right) P_{\text {byte }}^{9}\left(1-P_{\text {byte }}\right)^{200-9}
$$

Due to the small $P_{\text {byte, }}$, for each byte in error, only a single bit is likely to be wrong. Thus, the fraction of bits in error in the codeword is roughly $9 / 200 / 8=5.6 \cdot 10^{-3}$. Therefore, in order to achieve a target bit-error rate (BER) of $10^{-7}, P_{\text {byte }}$ must satisfy

$$
10^{-7}=\left(\begin{array}{c}
200 \\
9
\end{array}\right) P_{\text {byte }}^{9}\left(1-P_{\text {byte }}\right)^{191} 5.6 \cdot 10^{-3}
$$

or $P_{\text {byte }}=0.0065$. Taking the first term of the binomial expansion $P_{\text {byte }}=8 P_{b}\left(1-P_{\text {bit }}\right)^{8} \approx 8 P_{\text {bit }}$, the probability of bit error should be $P_{\text {bit }}=P_{\text {byte }} / 8=0.0065 / 8=8.1 \cdot 10^{-4}$ in order to attain an overall BER $10^{-7}$ after the decoding of the RS code. The signal-to-noise (SNR) gap for quadrature amplitude modulation (QAM) for $P_{\mathrm{bit}}=8.1 \cdot 10^{-4}$ is found by noticing that $2 Q(10.5 \mathrm{~dB})=8.1 \cdot 10^{-4}$. Hence, the coding gain is equal to $14.5 \mathrm{~dB}-10.5 \mathrm{~dB}=4.0 \mathrm{~dB}$. Table I summarizes the coding gains of the RS code for small $P_{\text {byte }}$ under various system conditions. 
TABLE I

PARAMETERS OF RS FEC FOR ADSL SYSTEMS

\begin{tabular}{|c|c|c|c|c|}
\hline \multicolumn{2}{|c|}{ Target data rate } & $608 \mathrm{kbps}$ & $1.216 \mathrm{Mbps}$ & $2.048 \mathrm{Mbps}$ \\
\hline \multicolumn{2}{|c|}{ Number of bytes per DMT } & 23 & 42 & 68 \\
\hline \multirow{5}{*}{ Fast mode } & DMT symbols per RS & 1 & 1 & 1 \\
\hline & RS codeword length & 23 bytes & 42 bytes & 68 bytes \\
\hline & Parity length & 4 bytes & 4 bytes & 6 bytes \\
\hline & RS coding gain & $3.0 \mathrm{~dB}$ & $2.78 \mathrm{~dB}$ & $3.2 \mathrm{~dB}$ \\
\hline & Coding overhead & $128 \mathrm{kbps}$ & $128 \mathrm{kbps}$ & $192 \mathrm{kbps}$ \\
\hline \multirow{5}{*}{ Interleave Mode } & DMT symbols per RS & 8 & 4 & 2 \\
\hline & RS Codeword length & 200 bytes & 184 bytes & 152 bytes \\
\hline & Parity length & 16 bytes & 16 bytes & 16 bytes \\
\hline & RS coding gain & $4.00 \mathrm{~dB}$ & $4.03 \mathrm{~dB}$ & $4.16 \mathrm{~dB}$ \\
\hline & Coding overhead & $64 \mathrm{kbps}$ & $128 \mathrm{kbps}$ & $256 \mathrm{kbps}$ \\
\hline
\end{tabular}

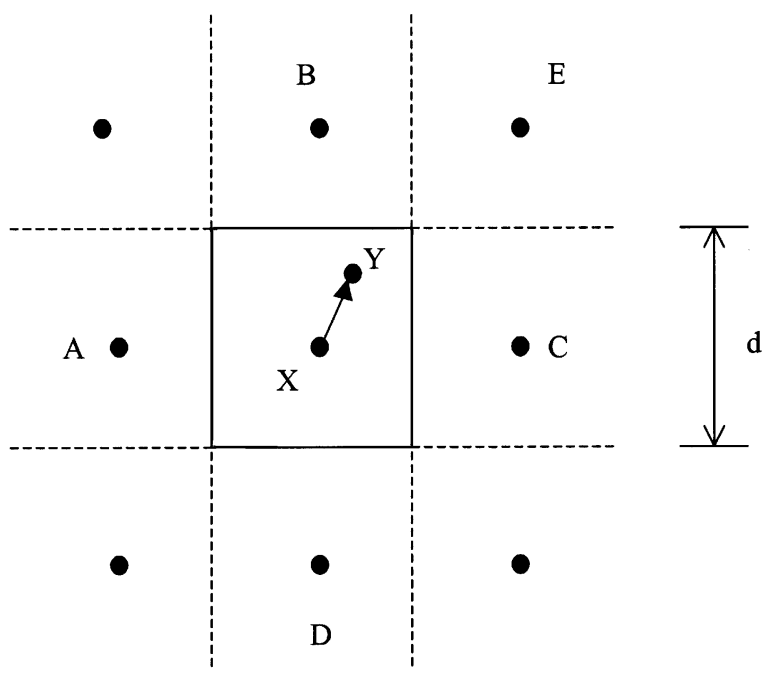

Fig. 1. Probability of error calculation.

\section{ADSL PERFORMANCE With IMPULSE NOISE}

Suppose that a tone of the DMT symbol is hit by a deterministic impulse shown in Fig. 1 as an arrow from the original constellation point $X$ to the new location $Y$. If $Y$ is outside the decision boundary, an error is almost certain to occur, so $P_{e}=1$, and $P_{\mathrm{bit}}=1 / 2$. If $Y$ is outside the decision boundary, a symbol error is almost certain to occur, and $P_{\mathrm{bit}}=1 / 2 \sum_{j} Q\left(d_{j} / 2 \sigma\right)$, where $d_{j}$ 's are the distances between $Y$ and the boundaries of the decoding regions of its neighbors $A, B, C, D$, and $E$. Strictly speaking, summing the probability is only valid when the dimensions are orthogonal. The above formula is a union bound. The factor $1 / 2$ accounts for the conversion between the probability of symbol error and the probability of bit error. It represents a worst-case scenario, and a practical system with Gray code bit mapping may have lower probability of bit error.

In an uncoded system, the probability of bit error of the entire DMT symbol can be found by averaging the probability of bit error in each tone: $P_{\mathrm{bit}, \mathrm{DMT}}=\sum_{i} b_{i} P_{\mathrm{bit}}(i) / \sum_{i} b_{i}$, where $P_{\mathrm{bit}}(i)$ is the probability of bit error and $b_{i}$ the number of bits of the $i$ th tone.

In a coded system, in order to evaluate the expected number of error bytes in an RS codeword, the probability of byte error

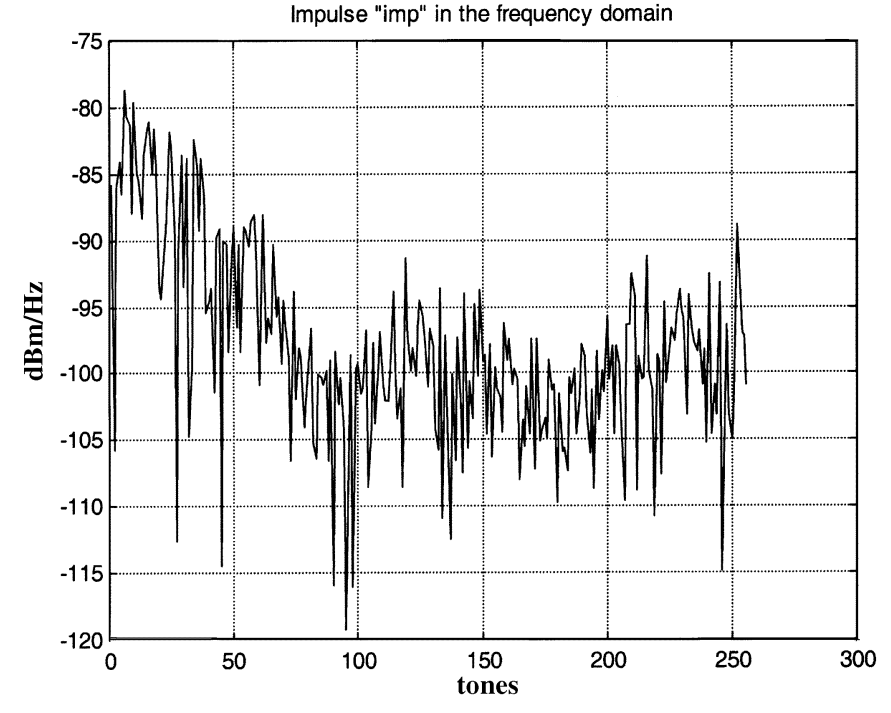

Fig. 2. Frequency-domain plot of the impulse "imp."

is first computed as $P_{\text {byte }}=1-\prod_{i=1}^{8}\left(1-P_{\text {bit }}(i)\right)$. Since the byte boundaries do not necessarily coincide with the tone boundaries, the probabilities of bit error $P_{\mathrm{bit}}(i)$ for each bit may be different. In the absence of coding, the expected number of error bytes is just the sum $\sum_{k} P_{\text {byte }}(k)$, summed over all bytes in the codeword. When an RS code is used, the codewords with fewer than nine errors are corrected. Let $P_{\text {error }}(n)$ denote the probability that there are $n$ error bytes in an RS codeword. Then, the average number of byte errors is computed as follows: $\sum_{n>8} n P_{\text {error }}(n)=\sum_{n} n P_{\text {error }}(n)-\sum_{n<8} n P_{\text {error }}(n)=$ $\sum_{k} P_{\text {byte }}(k)-\sum_{n \leq 8} n P_{\text {error }}(n)$. To reduce the computational effort, $P_{\text {error }}(n)$ can be very closely approximated by selecting only the large probability terms which typically correspond to the few bytes with large $P_{\text {byte }}(k)$.

\section{Simulation Results}

The performance of both coded and uncoded systems is first evaluated for three representative impulse samples (named "imp," "ex," and "raf"). The "imp" impulse lasts for $500 \mu \mathrm{s}$ (i.e., is two DMT symbols long) and has the highest peak voltage. Its frequency domain plot is given in Fig. 2 as an example. "ex" has slightly smaller peak voltage, and only lasts for one DMT symbol, whereas "raf" has the smallest peak voltage, and is about 2.5 DMT symbols long. The average BER for the uncoded system over a DMT symbol at the maximum reaches for each margin value and for three different noise models is shown in Table II. SC1 and SC2 are proprietary crosstalk models for the network of France Télécom. As expected, the BER largely depends on the duration of the impulse. The BER also depends on the noise model. Impulse noise will have a more severe effect on systems designed for AWGN channels compared to channels with crosstalk. This is due to the fact that a system designed for a crosstalk environment has to be more robust, since the power of crosstalk is higher compared to AGWN. In general, the service range of a system designed to cope with crosstalk is smaller, and the distance between the constellation points larger. Consequently, it is more immune to impulse noise as well. Since the impulse simulation is 
TABLE II

PROBABILITY OF BIT ERROR FOR EACH OF THE THREE IMPULSES (UNCODED)

\begin{tabular}{|c|c|c|c|c|c|c|c|c|c|c|}
\hline & Rate & \multicolumn{3}{|c|}{ 608kbps } & \multicolumn{3}{|c|}{$1.216 \mathrm{Mbps}$} & \multicolumn{3}{|c|}{$2.048 \mathrm{Mbps}$} \\
\hline Impulse & Noise & AWGN & $\mathrm{SC} 1$ & $\mathrm{SC} 2$ & AWGN & $\mathrm{SC} 1$ & SC2 & AWGN & $\mathrm{SC1}$ & SC2 \\
\hline "imp" & $0 \mathrm{~dB}$ & 0.5 & 0.5 & 0.5 & 0.5 & 0.5 & 0.5 & 0.5 & 0.5 & 0 \\
\hline high peak & $6 \mathrm{~dB}$ & 0.5 & 0.5 & 0.3 & 0.5 & 0.4 & 0.25 & 0.5 & 0.3 & 0.25 \\
\hline $2 \mathrm{DMT}$ & $12 \mathrm{~dB}$ & 0.5 & 0.2 & 0.7 & 0.5 & 0.2 & 0.7 & 0.5 & 0.1 & 0.07 \\
\hline "ex" & $0 \mathrm{~dB}$ & 0.5 & 0.5 & 0.5 & 0.5 & 0.5 & 0.5 & 0.5 & 0.5 & 0.5 \\
\hline smaller peak & $6 \mathrm{~dB}$ & 0.5 & 0.4 & 0.3 & 0.5 & 0.35 & 0.3 & 0.5 & 0.35 & 0.2 \\
\hline $1 \mathrm{DMT}$ & $12 \mathrm{~dB}$ & 0.5 & 0.35 & 0.25 & 0.5 & 0.3 & 0.2 & 0.5 & 0.3 & 0.2 \\
\hline "raf" & $0 \mathrm{~dB}$ & 0.5 & 0.5 & 0.5 & 0.5 & 0.5 & 0.5 & 0.5 & 0.5 & 0.5 \\
\hline smallest peak & $6 \mathrm{~dB}$ & 0.4 & 0.08 & 0.05 & 0.3 & 0.8 & 0.05 & 0.3 & 0.1 & 0.05 \\
\hline $2.5 \mathrm{DMT}$ & $12 \mathrm{~dB}$ & 0.3 & 0.019 & 0 & 0.25 & 0.038 & 0.005 & 0.22 & 0.13 & 0.007 \\
\hline
\end{tabular}

TABLE III

EXPected Number of ERror Bytes at MaXimum Reaches as a Function of InterleaVer Depth, Impulse, Margin, Crosstalk Model, and TARget Rate

\begin{tabular}{|c|c|c|c|c|c|c|c|c|c|c|}
\hline \multirow{3}{*}{\multicolumn{2}{|c|}{$\begin{array}{c}\text { Coding } \\
\text { Margin }\end{array}$}} & \multicolumn{3}{|c|}{ Impulse "imp" } & \multicolumn{3}{|c|}{ Impulse "ex" } & \multicolumn{3}{|c|}{ Impulse "raf" } \\
\hline & & \multicolumn{9}{|c|}{ FEC, no interleaving $(D=0)$} \\
\hline & & $0 \mathrm{~dB}$ & $6 \mathrm{~dB}$ & $12 \mathrm{~dB}$ & $0 \mathrm{~dB}$ & $6 \mathrm{~dB}$ & $12 \mathrm{~dB}$ & $0 \mathrm{~dB}$ & $6 \mathrm{~dB}$ & $12 \mathrm{~dB}$ \\
\hline \multirow{3}{*}{$\begin{array}{c}608 \\
\text { kbps }\end{array}$} & AWGN & 74 & 73 & 71 & 48 & 47 & 47 & 84 & 81 & 72 \\
\hline & $\mathrm{SC} 1$ & 72 & 59 & 32 & 46 & 39 & 32 & 73 & 34 & 15 \\
\hline & SC2 & 44 & 35 & 21 & 34 & 33 & 29 & 39 & 25 & 2 \\
\hline \multirow{3}{*}{$\begin{array}{c}1.2 \\
\text { Mbps }\end{array}$} & AWGN & 133 & 131 & 129 & 86 & 85 & 86 & 149 & 138 & 118 \\
\hline & $\mathrm{SC} 1$ & 125 & 92 & 55 & 81 & 66 & 54 & 109 & 57 & 33 \\
\hline & $\mathrm{SC} 2$ & 80 & 64 & 31 & 63 & 62 & 48 & 74 & 41 & 9 \\
\hline \multirow{3}{*}{$\begin{array}{c}2.0 \\
\text { Mbps }\end{array}$} & AWGN & 216 & 213 & 205 & 139 & 138 & 139 & 243 & 225 & 190 \\
\hline & $\mathrm{SC} 1$ & 187 & 128 & 60 & 124 & 110 & 78 & 144 & 99 & 20 \\
\hline & $\mathrm{SC} 2$ & 134 & 100 & 46 & 107 & 97 & 71 & 130 & 61 & 16 \\
\hline \multicolumn{2}{|c|}{ Coding } & \multicolumn{9}{|c|}{ FEC with Interleaving $D=8$} \\
\hline \multicolumn{2}{|c|}{ Margin } & $0 \mathrm{~dB}$ & $6 \mathrm{~dB}$ & $12 \mathrm{~dB}$ & $0 \mathrm{~dB}$ & $6 \mathrm{~dB}$ & $12 \mathrm{~dB}$ & $0 \mathrm{~dB}$ & $6 \mathrm{~dB}$ & $12 \mathrm{~dB}$ \\
\hline \multirow{3}{*}{$\begin{array}{c}608 \\
\text { kbps }\end{array}$} & AWGN & 75 & 60 & 56 & 28 & 0 & 0 & 90 & 77 & 64 \\
\hline & SC1 & 74 & 21 & 0 & 18 & 0 & 0 & 79 & 1 & 0 \\
\hline & $\mathrm{SC} 2$ & 7 & 0 & 0 & 2 & 0 & 0 & 2 & 0 & 0 \\
\hline \multirow{3}{*}{$\begin{array}{c}1.2 \\
\text { Mbps }\end{array}$} & AWGN & 138 & 126 & 124 & 81 & 72 & 73 & 155 & 135 & 119 \\
\hline & $\mathrm{SCl}$ & 132 & 89 & 23 & 77 & 56 & 31 & 118 & 29 & 2 \\
\hline & $\mathrm{SC} 2$ & 83 & 37 & 0 & 60 & 39 & 4 & 74 & 2 & 0 \\
\hline \multirow{3}{*}{$\begin{array}{c}2.0 \\
\text { Mbps }\end{array}$} & AWGN & 225 & 216 & 202 & 148 & 140 & 137 & 250 & 216 & 176 \\
\hline & $\mathrm{SC} 1$ & 199 & 121 & 38 & 135 & 100 & 62 & 157 & 93 & 0 \\
\hline & SC2 & 140 & 97 & 6 & 113 & 87 & 57 & 134 & 48 & 0 \\
\hline \multicolumn{2}{|c|}{ Coding } & \multicolumn{9}{|c|}{ FEC with Interleaving $D=32$} \\
\hline \multicolumn{2}{|c|}{ Margin } & $0 \mathrm{~dB}$ & $6 \mathrm{~dB}$ & $12 \mathrm{~dB}$ & $0 \mathrm{~dB}$ & $6 \mathrm{~dB}$ & $12 \mathrm{~dB}$ & $0 \mathrm{~dB}$ & $6 \mathrm{~dB}$ & $12 \mathrm{~dB}$ \\
\hline \multirow{3}{*}{$\begin{array}{c}608 \\
\text { kbps }\end{array}$} & AWGN & 9 & 0 & 0 & 8 & 0 & 0 & 13 & 0 & 0 \\
\hline & $\mathrm{SC} 1$ & 9 & 0 & 0 & 8 & 0 & 0 & 13 & 0 & 0 \\
\hline & SC2 & 7 & 0 & 0 & 6 & 0 & 0 & 9 & 0 & 0 \\
\hline \multirow{3}{*}{$\begin{array}{c}1.2 \\
\text { Mbps }\end{array}$} & AWGN & 8 & 0 & 0 & 8 & 0 & 0 & 16 & 0 & 0 \\
\hline & $\mathrm{SC} 1$ & 8 & 0 & 0 & 8 & 0 & 0 & 15 & 0 & 0 \\
\hline & $\mathrm{SC} 2$ & 8 & 0 & 0 & 6 & 0 & 0 & 12 & 0 & 0 \\
\hline \multirow{3}{*}{$\begin{array}{c}2.0 \\
\text { Mbps }\end{array}$} & AWGN & 209 & 62 & 27 & 6 & 16 & 16 & 14 & 0 & 0 \\
\hline & $\mathrm{SCl}$ & 81 & 0 & 0 & 7 & 0 & 0 & 14 & 0 & 0 \\
\hline & SC2 & 7 & 0 & 0 & 13 & 0 & 0 & 16 & 0 & 0 \\
\hline \multicolumn{2}{|c|}{ Coding } & & & & $\mathrm{C}$ with & Interle & ing $D=$ & & & \\
\hline & rgin & $0 \mathrm{~dB}$ & $6 \mathrm{~dB}$ & $12 \mathrm{~dB}$ & $0 \mathrm{~dB}$ & $6 \mathrm{~dB}$ & $12 \mathrm{~dB}$ & $0 \mathrm{~dB}$ & $6 \mathrm{~dB}$ & $12 \mathrm{~dB}$ \\
\hline & AWGN & 18 & 0 & 0 & 13 & 0 & 0 & 18 & 0 & 0 \\
\hline khns & $\mathrm{SC} 1$ & 17 & 0 & 0 & 13 & 0 & 0 & 17 & 0 & 0 \\
\hline & $\mathrm{SC} 2$ & 11 & 0 & 0 & 9 & 0 & 0 & 10 & 0 & 0 \\
\hline & AWGN & 16 & 0 & 0 & 16 & 0 & 0 & 16 & 0 & 0 \\
\hline Mhns & $\mathrm{SC} 1$ & 16 & 0 & 0 & 15 & 0 & 0 & 16 & 0 & 0 \\
\hline Nopos & $\mathrm{SC} 2$ & 14 & 0 & 0 & 12 & 0 & 0 & 15 & 0 & 0 \\
\hline & AWGN & 14 & 0 & 0 & 14 & 0 & 0 & 14 & 0 & 0 \\
\hline Mbps & SC1 & 14 & 0 & 0 & 14 & 0 & 0 & 14 & 0 & 0 \\
\hline Miops & $\mathrm{SC} 2$ & 16 & 0 & 0 & 16 & 0 & 0 & 16 & 0 & 0 \\
\hline
\end{tabular}



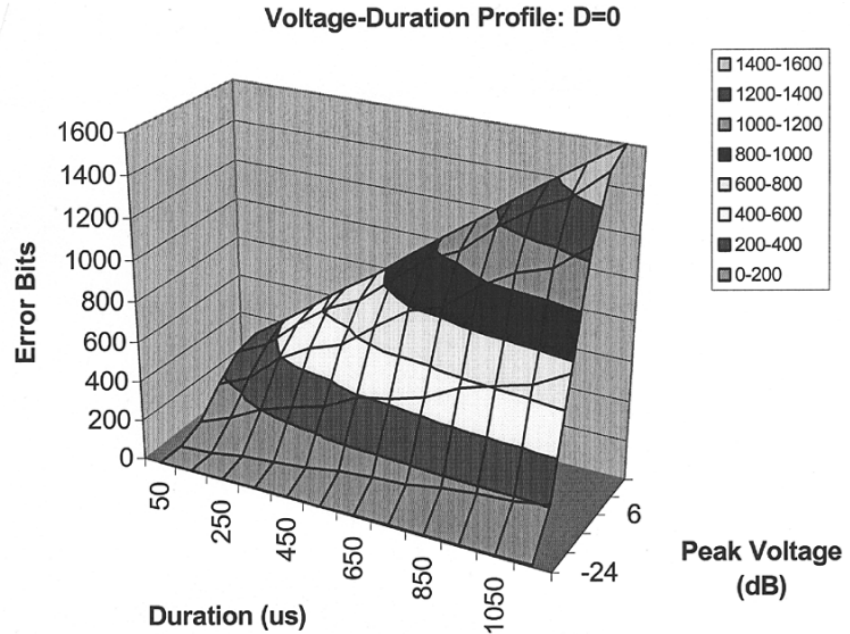

Voltage-Duration Profile: $D=32$

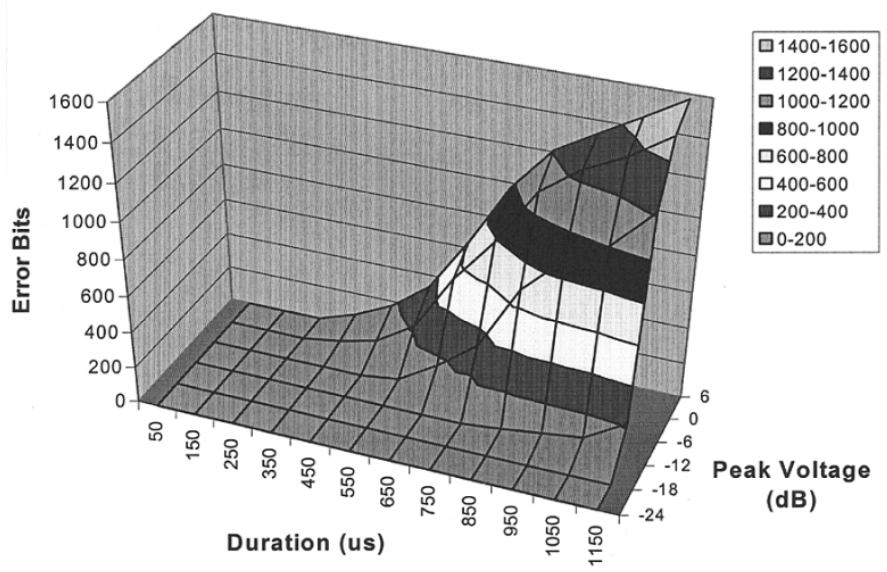

Fig. 3. Voltage-duration profile for interleaver depth $D=0$ and $D=32$.

performed at the maximum reaches, systems designed for a crosstalk environment appear to be more robust against impulse noise. Table III presents the coded system performance with and without interleaving for the three representative impulses. It is assumed that impulses occur infrequently so that no RS codeword can ever contain bytes corrupted by two different impulses. In all three cases, complete protection again impulse attacks is obtained with a combination of 6-dB noise margin, and an RS code with interleaver depth of 64 . Neither noise margin, nor coding, alone is adequate.

Next, the impulse "raf" is used to illustrate how the performance of ADSL is affected by impulse characteristics. The number of error bits is plotted against the impulse peak voltage and the impulse duration. The original impulse is $600 \mu \mathrm{s} \mathrm{long.}$ Shorter impulses are created by truncating, and longer impulses by concatenating replicas of "raf." Impulses with different peak voltages are created by scaling. The peak voltage axis is in decibels, and $0 \mathrm{~dB}$ corresponds to the peak voltage of the original impulse. The results are plotted in Fig. 3. AWGN noise is assumed, and a 6- $\mathrm{dB}$ margin is included. The maximum ranges for the $2.048 \mathrm{Mb} / \mathrm{s}$ system are used. It is interesting to observe that in the fast mode, the number of error bits increases linearly with duration. In the interleaved mode, the number

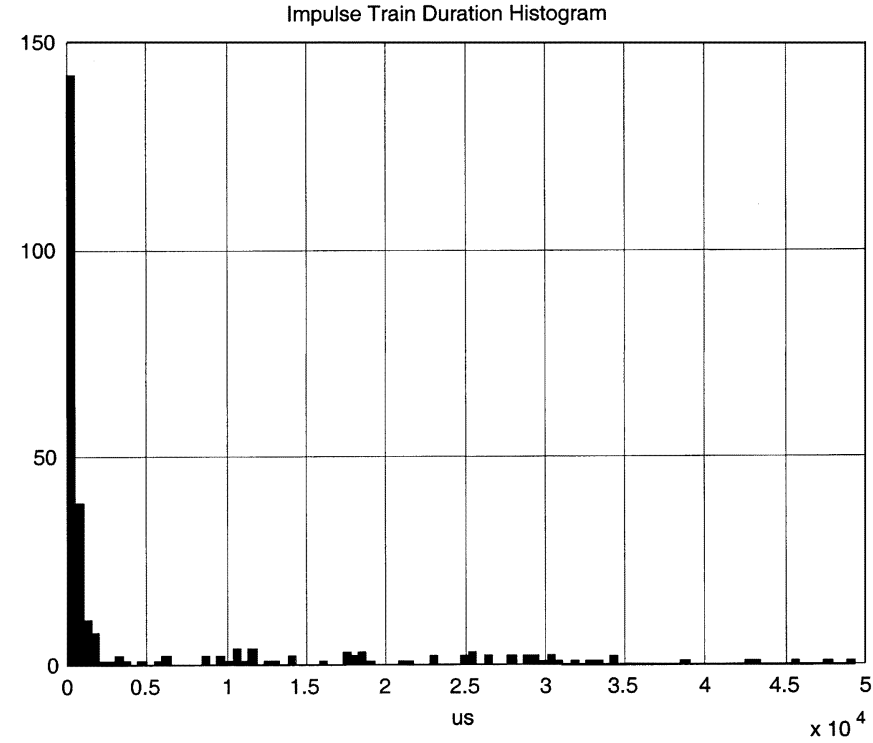

Fig. 4. Duration histogram of the impulses used for the simulations.

of error bits is suppressed for short duration impulses, but eventually grows linearly again as the interleaver breaks down for longer impulses.

Finally, the effect of impulse trains, i.e, bursts of subimpulses close to each other, is characterized using the notion of error seconds. In this study, all impulse trains are less than 1/16 s long. So, each impulse train can cause, at most, one error second. Therefore, determining the number of error seconds is equivalent to determining the number of impulse trains that cannot be corrected by FEC.

In the simulations, 269 impulse trains occurring over a two-day period were used. The duration histogram of the impulse trains is plotted in Fig. 4. Most impulse trains are less than $3 \mathrm{~ms}$ long (or 12 DMT symbols), but the longest ones can last up to $50 \mathrm{~ms}$ (or $256 \mathrm{DMT}$ symbols). The number of error seconds is summarized in Table IV. No interleaving is used in the fast mode. In the medium and long delay modes, 16 and 64 DMT symbols, respectively, are interleaved.

The above results clearly illustrate a number of tradeoffs. First of all, better impulse protection requires longer interleaving delays. Secondly, for a given delay, a higher margin system is able to withstand a larger number of impulses. However, the noise margin comes in the expense of maximum reach. Hence, there is a tradeoff among interleaving delay and maximum reach. As an example, Fig. 5 summarizes those tradeoffs for a $2.048 \mathrm{Mb} / \mathrm{s}$ system under a moderate amount of crosstalk (SC1).

\section{CONCLUSIONS}

Typical impulses occurring on ADSL lines are 20-40 dB larger than either AWGN or near-end crosstalk, and they can be several DMT symbols long. Such significant disturbance can destroy the ADSL performance completely when no FEC is used. Noise margin of 6 or $12 \mathrm{~dB}$ alone is not sufficient to protect ADSL from impulse noise. With FEC, a size-64 interleaver and $6 \mathrm{~dB}$ of noise margin, almost complete protection against an isolated impulse (of duration up to $500 \mu \mathrm{s}$ ) can be obtained regardless of its peak voltage. However, real ADSL 
TABLE IV

NUMBER OF ERROR SECONDS IN TwO DAYS

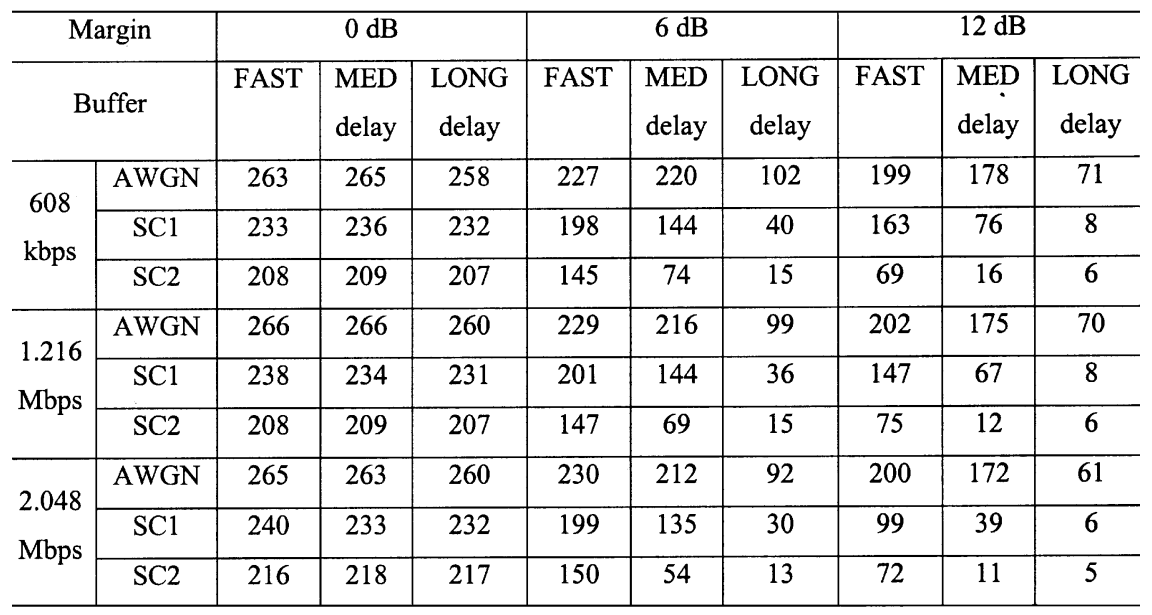

Tradeoff between Margin, Delay and Impulse Protection (2.048Mbps, SC1)

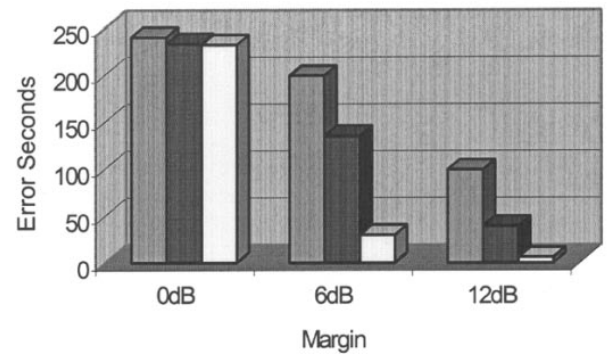

\begin{tabular}{|l|}
\hline a Fast Mode \\
aedium Delay \\
口Long Delay \\
\hline
\end{tabular}

Fig. 5. Tradeoff between margin, delay, and impulse protection.

deployment often experiences extended impulse trains that will occasionally break the FEC code, even when a 6-dB margin and an interleaving depth of 64 are used. The tradeoff among impulse protection, margin, and delay is characterized. These findings are useful for ADSL deployment planning.

\section{REFERENCES}

[1] "Draft New Recommendation G.992.1: Asymmetrical Digital Subscriber Line (ADSL) Transceivers (ex G.dmt)," International Telecommunication Union (ITU), Geneva, Switzerland, 1999.

[2] I. Mann, S. McLaughlin, W. Henkel, R. Kirkby, and T. Kessler, "Impulse generation with appropriate amplitude, length, interarrival, and spectral characteristics," IEEE J. Select. Areas Commun., vol. 20, pp. 901-912, June 2002.

[3] W. Henkel, T. Kessler, and H. Y. Chung, "Coded 64-CAP ADSL in an impulse-noise environment-Modeling of impulse noise and first simulation results," IEEE J. Select. Areas Commun., vol. 13, pp. 1611-1621, Dec. 1995.

[4] D. B. Levey and S. McLaughlin, "Calculating error-free seconds in xDSL systems corrupted by impulse noise," IEEE Commun. Lett., vol. 5, pp. 319-321, July 2001.

[5] J. W. Modestino and D. H. Sargrad, "Use of coding to combat impulse noise on digital subscriber loops," IEEE Trans. Commun., vol. 36, pp. 529-537, May 1988.

[6] N. Nedev, S. McLaughlin, D. Laurenson, and R. Daley, "ATM cell error performance of xDSL under impulse noise," in Proc. Int. Conf. Communications, vol. IV, Helsinki, Finland, June 2001, pp. 1254-1258.

[7] S. B. Wicker, Error Control Systems for Digital Communication and Storage. Englewood Cliffs, NJ: Prentice-Hall, 1995. 